Received: 2013.09 .09

Accepted: 2013.11.08

Published: 2014.02.19

\title{
Comparison of single-dose and repeated levosimendan infusion in patients with acute exacerbation of advanced heart failure
}

Authors' Contribution: Study Design A Data Collection B Statistical Analysis C Data Interpretation D Manuscript Preparation E Literature Search F Funds Collection G
ABCDEF 1 Abdurrahman Tasal

BDF 2 Mesut Demir

BCD 2 Mehmet Kanadasi

BCD 1 Ahmet Bacaksiz

BCD 1 Mehmet Akif Vatankulu

$B C D$ Durmus Yıldıray Sahin

BCD 2 Rabia Akıllı Eker

BCD 2 Abdi Bozkurt

$B C D 2$ Esmeray Acarturk
Corresponding Author: Source of support:
1 Department of Cardiology, Bezmialem Vakif University, Istanbul, Turkey 2 Department of Cardiology, Cukurova University, Adana, Turkey 3 Department of Cardiology, Adana Numune Education and Research Hospital, Adana, Turkey

Background: Levosimendan (LS) is a novel inodilator that improves cardiac performance, central hemodynamics, and symptoms of patients with decompensated chronic heart failure. The aim of this study was to compare the effects of single and repeated LS infusion on left ventricular performance, biomarkers, and neurohormonal activation in patients with acute heart failure.

Material/Methods: Twenty-nine consecutive patients with acute exacerbation of advanced heart failure were included in this study. LS was initiated as a bolus of $6 \mu \mathrm{g} / \mathrm{kg}$ followed by a continuous infusion of $0.1 \mu \mathrm{g} / \mathrm{kg} / \mathrm{min}$ for 24 hours in both groups who received intravenous single and repeated (baseline and at 1 and 3 months) treatment. Physical examination, echocardiography, and biochemical tests (brain natriuretic peptide, tumour necrosis factor- $\alpha$, interleukin-1 $\beta, 2$, and 6 ) were performed before treatment and on 3 day of the treatment. The last evaluation was performed at 6 month after the baseline treatment.

Results: $\quad$ Twenty male and 9 female patients with mean age of $60.2 \pm 7.4$ years were included in this study. A significant improvement in New York Heart Association functional status and myocardial performance index was detected only in the repeated LS treated patients at 6 month compared to the pretreatment status $(p=0.03$ and $p<0.001$; respectively). In addition, a significant decrease in brain natriuretic peptide $(p<0.01)$ and plasma interleukin- 6 $(p=0.05)$ levels were also achieved only in patients who were given repeated LS.

Conclusions: Our study showed that repeated LS treatment is more effective compared to the single dose LS treatment in improving clinical status, hemodynamic and laboratory parameters in patients with acute exacerbation of advanced heart failure.

MeSH Keywords: $\quad$ Levosimendan • Interleukin-6• Heart Failure

Full-text PDF: http://www.medscimonit.com/download/index/idArt/889767

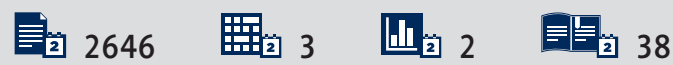




\section{Background}

Acute decompensation of chronic heart failure (HF) is a lifethreatening situation with a short-term mortality of approximately $30 \%$ [1]. Intravenous positive inotropic agents play an important role in the treatment of acute decompensation in patients with systolic HF. Although traditional inotropic agents such as, $\beta$-adrenergic agonists and phosphodiesterase inhibitors appear effective for short-term improvement of symptoms, previous studies demonstrated that these agents increase morbidity and mortality by elevating intracellular cyclic adenosine monophosphate (cAMP) and calcium ( $\left.\mathrm{Ca}^{+2}\right)$ levels [2,3]. Despite optimum traditional treatment strategies, frequent hospitalization due to acute decompensation leads to worsening of the disease [4].

Levosimendan (LS) is a $\mathrm{Ca}^{+2}$ sensitizer for the treatment of decompensated $\mathrm{HF}$ that increases troponin- $\mathrm{C}$ sensitivity against cytoplasmic $\mathrm{Ca}^{+2}$ without any modification of intracellular $\mathrm{Ca}^{+2}$ density [5]. LS's sensitizing effect is dependent on the $\mathrm{Ca}^{+2}$ concentration, thus its affinity to cardiac myofilaments increases during systole and improves cardiac contractility [6]. During diastole, due to a decrease in the $\mathrm{Ca}^{+2}$ concentration, $\mathrm{LS}$ does not cause deterioration in diastolic functions [6]. LS improves cardiac contractility, increases cardiac output and stroke volume, and decreases pulmonary capillary wedge pressure, pulmonary artery pressure, and systemic and vascular resistance [7-9].

Cardiac remodelling is an important mechanism in the development and progression of HF [10]. Neurohormonal activation and proinflammatory cytokines play key roles in this process [10]. In severe HF, despite optimum medical treatment, elevated plasma brain natriuretic peptide (BNP), and interleukin levels were reported to be independent risk factors for both morbidity and mortality [11]. LS treatment decreased the BNP and interleukin levels [12]. Although previous studies displayed beneficial effects of both single-dose and repeated LS treatment on hemodynamics, symptoms, and inflammatory markers in patients with acute decompensated HF, there was no consensus on the frequency of its use. No data in the current literature exist concerning optimal dosing of LS in patients with acute decompensated HF.

In this study, we researched the impact of single-dose versus repeated LS infusion on cardiac performance, immune reaction, and neurohormonal activation in patients with acute decompensated HF.

\section{Material and Methods}

\section{Patients}

The present study included patients hospitalized at Cukurova University Faculty of Medicine Cardiology Clinic upon diagnosis of acute decompensated HF, with an ejection fraction less than $35 \%$, and who were severely symptomatic (New York Heart Association [NYHA]-III/IV) although taking intravenous diuretic and vasodilator therapy. A total of 29 patients who met the inclusion criteria were included in the study and divided into 2 groups consecutively (1:1). A single-dose LS was given to patients in the first group and repeated LS was given to patients in the second group. The patients' clinical conditions and echocardiographic and laboratory findings were assessed at the start of the study and at 3 days and 6 months after treatment.

The following patients were excluded from the study:

1. Patients younger than 18 years of age.

2. Patients with restrictive or hypertrophic cardiomyopathy.

3. Patients with ischemic chest pain.

4. Patients who died during initial hospitalization.

5. Patients with continuous ventricular tachycardia (VT) or ventricular fibrillation in the course of hospitalization.

6. Patients with a heart rate greater than $120 / \mathrm{min}$.

7. Patients with a systolic blood pressure in the decubitus position less than $85 \mathrm{~mm} \mathrm{Hg}$

8. Patients with serious renal failure (creatine level $>2.5 \mathrm{gr} / \mathrm{dl}$ ) and severe liver failure

9. Patients unable to provide informed consent.

All subjects gave informed consent and the study was approved by the local ethics committee.

\section{Study protocol}

LS infusion in addition to standardized treatment was performed with a loading dose of $6 \mu \mathrm{g} / \mathrm{kg}$ for $10 \mathrm{~min}$ following a 24-h infusion of $0.1 \mu \mathrm{g} / \mathrm{kg} / \mathrm{min}$. LS was readministered in the first and third months to patients in the repeated treatment group. Clinical characteristics, echocardiographic measurements, and laboratory variables prior to treatment were evaluated after 1 dose and after 6 months. Patients in group 2 were hospitalized for LS infusion at 1 and 3 months. However, patients in group 1 were assessed at an outpatient clinic during follow-up without hemodynamic monitoring. The study endpoints were changes in clinical, biochemical, and echocardiographic variables at 6 month in both groups.

\section{Echocardiographic measurements}

Transthoracic echocardiographic examination was conducted on all patients using an Acuson Sequola C 256 model (Acuson Corporation, Mountain View, CA, USA) and a transducer with 3.5- $\mathrm{mHz}$ frequency along with ECG monitoring. All echocardiograms were performed by 2 echocardiographers blinded to the study. Examination was performed by finding convenient echocardiographic windows when the patient was in the decubitus position or lying on their left side. Left ventricular 
systolic and end-diastolic interior dimensions were measured from the obtained cross-sections and the left ventricular ejection fraction was calculated automatically. Moreover, left atrial systolic and end-diastolic sizes as well as septum and posterior wall thicknesses were measured from the same window. According to the Modified Simpson method, left ventricular systolic and end-diastolic volumes and ejection fraction (EF) were calculated [13].

Doppler velocity curves were recorded at a horizontal sweep speed of $100 \mathrm{~mm} / \mathrm{s}$ while the patient held their breath after exhalation. An average of 3 consecutive cycles was used for the analysis. Sample volume was placed between the tips of the mitral leaflets at apical 4 chambers and left ventricular filling variants were measured by pulse wave Doppler echocardiography technique. Based on the records obtained, left ventricle diastolic early (A wave) and late (E wave) peak velocities and mitral deceleration time (DT) were measured and the E/A ratio was calculated. Isovolumetric relaxation time (IVRT) obtained by placing the sample volume of continuous wave (CW) Doppler in the left ventricle outflow tract to simultaneously display the end of aortic ejection, and the onset of mitral inflow was measured as the interval in between. Time elapsed from the point where the mitral A wave finished to a point where the mitral E wave started (a), and left ventricle ejection time (b) was measured. Difference between the 2 measurements ( $a-b)$ was considered as the total of isovolumetric contract and IVRT. The myocardial performance index was then calculated using the $(a-b) / b$ formula.

During tissue Doppler measurements, specific attention was paid to ensuring that the ultrasonographic beams were parallel to the lateral and septal mitral annulus. Peak systolic mitral annuler (Sm), early diastolic (Em), and late diastolic (Am) velocities were measured with the sample volume placed at the junction of the LV wall and the mitral annulus of the septal and lateral myocardial segments from the 4-chamber view. The Sm velocity occurs during the ejection period, while the Em and Am velocities correspond to passive ventricular filling and atrial contraction. For assessment of left ventricular systolic function, Sm velocity was used.

Echocardiographic measurements were repeated in 5 subjects the next day by the same investigator. Additionally, transthoracic echocardiography was performed by a second observer for the same 5 patients after the first echocardiogram to assess the intra- and interobserver variability. Intraobserver and interobserver variability for lateral wall MPI were 0.03 and 0.06 , respectively.

\section{Laboratory measurements of inflammatory cytokines}

Blood samples were taken from all patients from the antecubital vein for the measurement of BNP, interleukin (IL)-1 $\beta$, IL-2,
IL-6, and tumour necrosis factor (TNF)- $\alpha$ before and after LS infusion and at 6-month follow-up. The samples were collected in tubes containing EDTA, then centrifuged at $1500 \mathrm{rpm}$ for a period of $5 \mathrm{~min}$ and stored at $-40^{\circ} \mathrm{C}$ until needed. After dissolution of serum samples, BNP level was measured by use of the electrochemiluminescent immunoassay method using Phoenix Pharmaceuticals (Burlingame, CA, USA) commercial kits, and IL-1 $\beta$, IL-2, IL- 6 , and TNF- $\alpha$ levels measured using the Biosource (Grand Island, NY, USA) commercial kit.

\section{Statistical analysis}

The study assay was performed using the SPSS 14.0 statistical software package. Continuous variations in group data are presented as means \pm standard deviation. Categorical data were compared by the use of the chi-square test. Subjects' data prior to treatment and at 3 days and 6 months post-treatment were compared using Wilcoxon's signed-rank test. Non-parametric data are expressed as percentages. A $p$-value $<0.05$ was considered to indicate statistical significance.

\section{Results}

Our study consisted of 29 patients divided into 2 groups (mean age $60.2 \pm 7.4$ years; 20 males, 9 females) with severe cardiac failure (NYHA-III/IV) who were administered a single dose or repeated dose of LS. Patient demographic characteristics are summarized in Table 1. Functional capacities of patients prior to treatment according to NYHA were similar in both groups (3.8 \pm 0.4 vs. $3.7 \pm 0.4, p=0.5)$. At the end of the follow-up period, a more robust recovery was observed in group $2(\triangle \mathrm{NYHA}$ $1.5 \pm 0.7$ vs. $0.9 \pm 0.6 ; p=0.03$; Figure 1 ).

The baseline echocardiographic indices were similar in both groups. The beneficial effects of LS on left ventricular parameters such as systolic volume, ejection fraction, myocardial performance index, and Sm was evident beginning on the third day after administration of the initial dose of LS (Table 2). Left ventricular systolic volume, ejection fraction, and myocardial performance index improvement continued to 6 months only in group 2 (Table 2 and Figure 2). In addition, left ventricular enddiastolic volume decreased during follow-up only in group 2 .

The patients' baseline BNP, IL-1, IL-2, IL-6, and TNF- $\alpha$ concentrations were elevated in both groups (Table 3). Seventy-two hours after LS infusion, a marked decline was detected in BNP, IL- 6 , and TNF- $\alpha$ concentrations. Decreased levels of BNP, IL-6, and TNF- $\alpha$ remained at 6 months only in group 2 .

All patients tolerated the treatment and were able to complete the infusion without adverse events. During the 6-month follow-up, no atrial fibrillation, ventricular tachycardia, or fibrillation developed 
Table 1. Demographic characteristics of patients prior to Levosimendan treatment.

\begin{tabular}{|c|c|c|c|}
\hline Demographic characteristics & Single dose $n=16$ & Repeated $n=13$ & $\mathbf{p}$ \\
\hline Age (year) & $59.6 \pm 7.2$ & $60.8 \pm 7.9$ & 0.72 \\
\hline Gender (female/male) & $6 / 10$ & $3 / 10$ & 0.41 \\
\hline Heart rate (pulse/min) & $85.5 \pm 5.8$ & $85.5 \pm 6.7$ & 0.86 \\
\hline Functional capasity (NYHA: III/IV) & $5 / 11$ & $4 / 9$ & 0.81 \\
\hline Systolic blood pressure $(\mathrm{mm} \mathrm{Hg})$ & $106.5 \pm 8.5$ & $105.7 \pm 8.6$ & 0.72 \\
\hline Diastolic blood pressure $(\mathrm{mm} \mathrm{Hg})$ & $70.9 \pm 8.2$ & $72.3 \pm 8.5$ & 0.48 \\
\hline Diabetes mellitus (\%) & $6 \quad(37.5)$ & $4 \quad(30.8)$ & 0.68 \\
\hline Hypertension (\%) & $8 \quad(50.0)$ & $9 \quad(69.2)$ & 0.23 \\
\hline Smoking (\%) & $5 \quad(31.3)$ & $8 \quad(61.5)$ & 0.22 \\
\hline Body surface area $\left(\mathrm{m}^{2}\right)$ & $1.8 \pm 0.16$ & $1.8 \pm 0.14$ & 0.87 \\
\hline Body mass index $\left(\mathrm{kg} / \mathrm{m}^{2}\right)$ & $23.8 \pm 2.0$ & $24.1 \pm 3.6$ & 0.71 \\
\hline Digoxin (\%) & $11 \quad(68)$ & $8 \quad(62)$ & 0.82 \\
\hline ACE inhibitor (\%) & $16(100)$ & $13(100)$ & 1.00 \\
\hline Diuretic (\%) & $16(100)$ & $13(100)$ & 1.00 \\
\hline Statin (\%) & $16(100)$ & $13(100)$ & 1.00 \\
\hline Amiodarone (\%) & $3 \quad(18)$ & $2 \quad(15)$ & 0.81 \\
\hline
\end{tabular}

LS - levosimendan, NYHA - New York Heart Association.

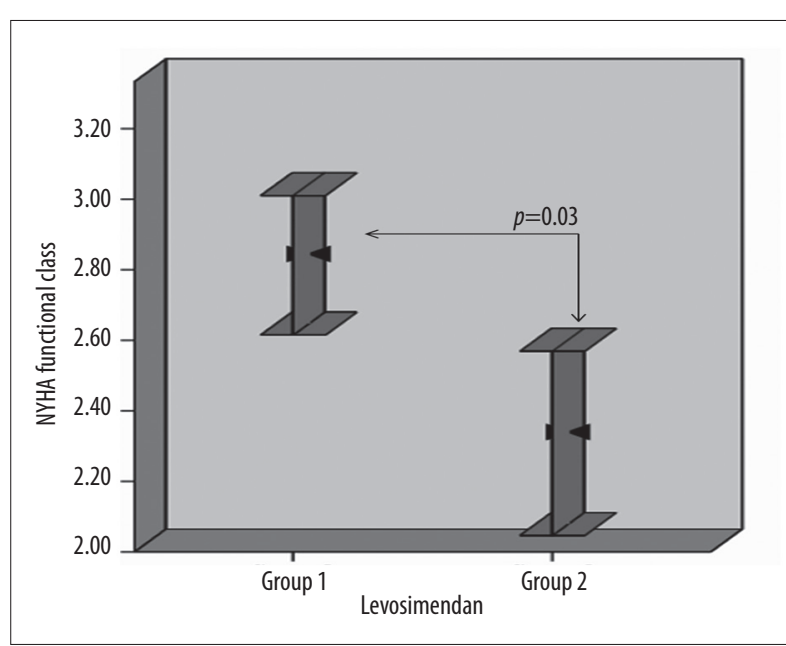

Figure 1. Comparison of functional capacities of the patients in both groups at 6 . month.

and no patient died. LS infusion was shortly interrupted 11 times (20\%) during infusion upon development of hypotension.

\section{Discussion}

This study showed that repeated LS treatment led to sustained improvement in functional status and left ventricular

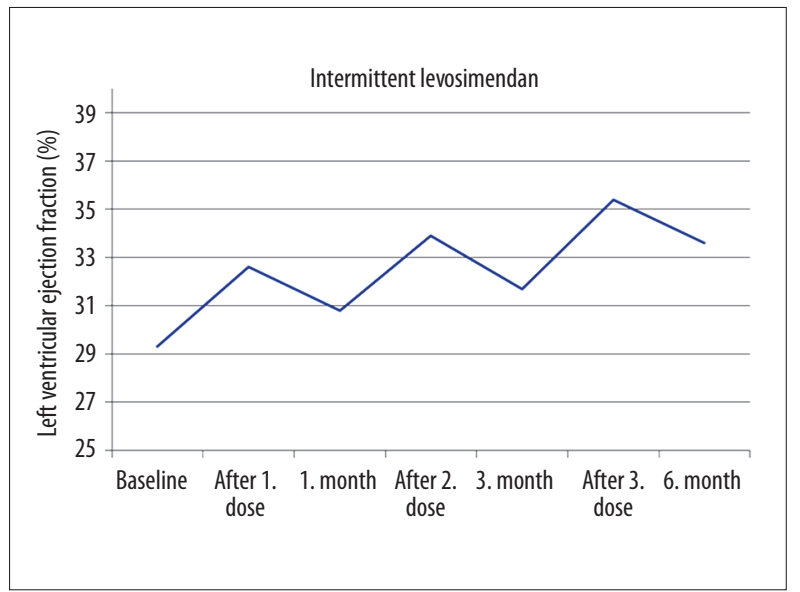

Figure 2. Beneficial effect of repeated levosimendan on echocardiographically measured left ventricular ejection fraction.

systolic functions at 6 months post-treatment compared to a single-dose infusion. Additionally, the treatment decreased the elevated levels of inflammatory cytokines considered as predictors of unfavorable outcome and mortality. A more intense therapy with repeated LS infusion in acute decompensated HF appeared as a reasonable option, especially for patients who could not remain stable without intravenous treatment. 
Table 2. Two dimensional echocardiographic findings comparision of two groups before and after first dose Levosimendan and at sixth month follow up.

\begin{tabular}{|c|c|c|c|c|c|c|}
\hline \multirow{2}{*}{$\begin{array}{c}\text { Echocardiographic } \\
\text { variables }\end{array}$} & \multicolumn{3}{|c|}{ Single dose } & \multicolumn{3}{|c|}{ Repeated } \\
\hline & Pre-treatment & $\begin{array}{l}\text { Post-treatment } \\
\text { day } 3\end{array}$ & 6. month & Pre-treatment & $\begin{array}{c}\text { Post-treatment } \\
\text { day } 3\end{array}$ & 6. month \\
\hline LVESV (ml) & $155.8 \pm 40.0$ & $138.1 \pm 40.0^{*}$ & $155.5 \pm 40.0^{\#}$ & $159.3 \pm 43.1$ & $142.4 \pm 64^{*}$ & $138 \pm 40.2^{\#, \S, t}$ \\
\hline LVEDV $(\mathrm{ml})$ & $212.5 \pm 34.9$ & $212.2 \pm 35.2$ & $212.6 \pm 35.4$ & $221.9 \pm 58.8$ & $221.7 \pm 58.3$ & $216.8 \pm 38.3^{\#, \S, t}$ \\
\hline MPI & $1.08 \pm 0.19$ & $0.88 \pm 0.16^{*}$ & $1.07 \pm 0.18^{\#}$ & $1.11 \pm 0.2$ & $0.94 \pm 0.2^{*}$ & $0.92 \pm 0.1^{\#, \S, \pm}$ \\
\hline $\mathrm{Sm}(\mathrm{cm} / \mathrm{s})$ & $8.3 \pm 0.8$ & $10.08 \pm 2.0^{*}$ & $8.2 \pm 1.0^{\#}$ & $8.03 \pm 1.0$ & $9.8 \pm 1.8^{*}$ & $9.6 \pm 1.01^{\#, S,}$ \\
\hline LVEF (\%) & $29.7 \pm 2.7$ & $33.4 \pm 3.2^{*}$ & $29.4 \pm 3.1^{\#}$ & $29.1 \pm 3.3$ & $32.4 \pm 4.4^{*}$ & $33.7 \pm 3.4^{\#, \S, \pm}$ \\
\hline
\end{tabular}

LVESV - left ventricular end sistolic volume; LVEDV - left ventricular end diastolic volume; Sm - peak systolic tissue Doppler velocity at lateral corner of mitral annulus; LVEF - left ventricular ejection fraction. * $p<0.05$ intra-group comparison of values before and after initial levosimendan infusion. ${ }^{*} p<0.05$ intra-group comparison of values after initial levosimendan infusion and values at 6 . month. $\S p<0.05$ intra-group comparison of values before initial levosimendan infusion and values at 6 . month. ${ }^{\ddagger} p<0.05$ inter-group comparison of values at 6 . month.

Table 3. Comparision of laboratory findings in both groups, pre-treatment, post-treatment and at sixth month follow up.

\begin{tabular}{|c|c|c|c|c|c|c|}
\hline & \multicolumn{3}{|c|}{ Single dose } & \multicolumn{3}{|c|}{ Repeated } \\
\hline & Pre-treatment & $\begin{array}{c}\text { Post-treatment } \\
\text { day } 3\end{array}$ & 6. month & Pre-treatment & $\begin{array}{c}\text { Post-treatment } \\
\text { day } 3\end{array}$ & 6. month \\
\hline BNP $(p g / m l)$ & $2015.3 \pm 871.3$ & $1282.6 \pm 827.2^{\star}$ & $1811.0 \pm 654.7^{\#}$ & $2306.4 \pm 775.1$ & $1326.5 \pm 702.8^{*}$ & $891.8 \pm 438.9^{\#, \S, \pm}$ \\
\hline $\mathrm{IL}-1 \beta(\mathrm{pg} / \mathrm{ml})$ & $0.93 \pm 1.3$ & $0.65 \pm 0.7$ & $0.7 \pm 0.8$ & $0.63 \pm 0.5$ & $0.62 \pm 0.9$ & $0.32 \pm 0.4$ \\
\hline IL-2 (pg/ml) & $0.24 \pm 0.3$ & $0.12 \pm 0.1$ & $0.20 \pm 0.2$ & $0.65 \pm 0.9$ & $0.38 \pm 0.1$ & $0.3 \pm 0.3$ \\
\hline IL-6 (pg/ml) & $14.6 \pm 9.8$ & $6.8 \pm 8.9^{\star}$ & $16.33 \pm 12.8^{\#}$ & $24.8 \pm 22.2$ & $9.7 \pm 6.0^{*}$ & $5.5 \pm 4.1^{\#, \S, \pm}$ \\
\hline TNF- $\alpha(p g / m l)$ & $6.1 \pm 3.9$ & $3.7 \pm 0.7^{\star}$ & $6.4 \pm 3.3^{\#}$ & $7.8 \pm 4.1$ & $4.7 \pm 3.2^{*}$ & $5.2 \pm 3.7^{\S, £}$ \\
\hline
\end{tabular}

BNP - B type natriuretic peptit; IL-1 $\beta$ - interleukin-1 beta; TNF- $\alpha$ - tumour necrosis factorr-alfa; IL- 6 - interleukin-6;

IL-2 - interleukin- $2{ }^{*} p<0.05$ intra-group comparison of values before and after initial levosimendan infusion. ${ }^{*} p<0.05$ intra-group comparison of values after initial levosimendan infusion and values at 6 . month. ${ }^{\S} p<0.05$ intra-group comparison of values before initial levosimendan infusion and values at 6 . month. ${ }^{ \pm} p<0.05$ inter-group comparison of values at 6 . month.

$\mathrm{HF}$ is a growing problem, especially as the elderly population is increasing, and for individuals and the general public with respect to re-hospitalization and frequent, prolonged hospitalization $[14,15]$. Although highly effective treatments including new drug therapies and devices have emerged, episodes of decompensation that require hospitalization still occur frequently $[1,16]$.

Cardiac remodeling is currently the accepted mechanism of the development and deterioration of HF $[10,17]$. The loss of cardiomyocytes through apoptosis and necrosis is an essential cellular pathway associated with excessive neurohormonal activation that leads to progression of LV remodeling is $[10,17]$. Elevated circulatory levels of proinflammatory cytokines (TNF- $\alpha$, IL-6, and IL-1 $\beta$ ) accelerate apoptosis in cardiac myocytes and endothelial cells by increasing oxidative stress and lead to suppression of myocardial contractility, all contributing to clinical and hemodynamic deterioration of $\mathrm{HF}$ $[18,19]$. Furthermore, despite optimum medical treatment, sustained high plasma levels of these factors are independent predictors of morbidity and mortality in patients with advanced HF [20]. Therefore, changes in BNP levels during treatment of decompensated chronic HF were strongly associated with early re-hospitalization and mortality [21]. Several studies suggested that LS leads to a marked decrease in BNP and proinflammatory levels and provides clinical improvement in these patients [22-24].

LS differs from traditional inotropic agents that display their positive inotropic effects by increasing intracellular $\mathrm{Ca}^{+2}$ concentrations, which may cause cardiomyocyte loss via the activation of apoptosis and necrosis $[25,26]$. LS has a dual mechanism of action: $\mathrm{Ca}^{+2}$-sensitization through binding to troponin $\mathrm{C}$ in the myocardium, and the opening of ATP-sensitive $\mathrm{K}^{+}$ 
channels in vascular smooth muscle [27]. These mechanisms evoke positive inotropy and vasodilation, which may result in improved cardiac output without increasing myocardial oxygen demand and impairing diastolic relaxation [28,29]. OR1896, an active metabolite of the drug, maintains the clinical response between 7 and 9 days after continuous intravenous infusion for $24 \mathrm{~h}$ [30,31]. Recovery based on echocardiographic, laboratory, and hemodynamic variables could be considered a result of a sustained decrease in mechanical overload [32].

The positive effects of both single-dose and repeated LS treatment in acute decompensated HF on hemodynamics, symptoms, and survival have been shown in various studies [32-35]. In a study by Mavrogeni et al. [36], monthly administration of LS for 6 months decreased left ventricle systolic and end-diastolic volume indices (16\% and $13 \%$, respectively), and increased left ventricle ejection fraction by $27 \%$. In an another study by Parissis et al. [12], 5 doses of repeated 24-h LS infusion were given at 3-week intervals, and patients were assessed 1 month after completion of the last dose. A decrease in cardiac volume and cardiac wall tension, an increase in left ventricle ejection fraction, and recovery in cardiac performance were observed with repeated LS therapy. Similarly, in our study, the beneficial effects of LS on left ventricular parameters such as systolic volume, ejection fraction, myocardial performance index, and Sm became clear as early as the third day after administration of the initial dose of LS in both groups. Improvement in left ventricular systolic volume, ejection fraction, and myocardial performance index continued for 6 months only in group 2. In addition, left ventricular enddiastolic volume, which was unchanged after the initial dose in both groups, decreased during follow-up only in group 2 . We speculate that delayed amelioration in diastolic measures could be explained by late onset of accumulated efficacy of LS on left ventricular geometry, which is in agreement with the results of a previous study [37].

One of the most important indicators of improved contractile functions with LS infusion is the increase in exercise capacity. In a tissue Doppler echocardiography study reported by Kasikcioglu et al. [38] there was a significant increase in the exercise capacity assessed by a 6-min walking test, similar to the increase of Sm after a single dose of LS. Most of the patients (70\%) allocated to repeated LS therapy were in NYHA class IV prior to treatment in our study. At the end of followup, functional status improved dramatically in $70 \%$ of the patients. However, a similar recovery was not found in patients who received a single dose of LS. Cardioprotective effects and

\section{References:}

1. Chen J, Normand ST, Wang Y, Krumholz HM: National and regional trends in heart failure hospitalization and mortality rates for Medicare beneficiaries. JAMA, 2011; 306: 1669-78 stronger protection against decompensation with LS could be the underlying mechanism of a more robust functional recovery with repeated dosing of LS.

Significant reductions in IL-6, BNP, and TNF- $\alpha$ levels were detected after the initial LS infusion in our study, which was in agreement with the results of previous studies [22-24]. The beneficial effects of LS on inflammatory cytokines disappeared at the end of 6 months in the single-dose group. However, the levels of these markers remained stable in the repeated LS group during follow-up. Clinical recovery and improved left ventricular function could eliminate the chronic inflammation process in these patients. Inconsistencies in IL-1 $\beta$ and IL- 2 levels may be explained by the small number of patients and intervening conditions such as infections, and transient hepatic or renal deterioration.

\section{Limitations}

The major limitation of this study was the small population size. Additionally, this was not a randomized and controlled study; no comparison was made with placebo or dobutamine. Patients in the single-dose group were not evaluated echocardiographically and biochemical measurements were not performed during the first and third months. Thus, the effects of repeated LS on biochemical and echocardiographic measures could not be compared. Results of this study should be confirmed in larger, prospective, randomized trials. Finally, the small sample size in this study precludes any valid subgroup analysis, thus we did not identify any confounders that may have interacted with treatment assignment and thus affect the outcome.

\section{Conclusions}

In this study we showed that repeated LS treatment in patients with severe cardiac failure when compared to singledose administration of LS, caused regulation and recovery of clinical symptoms and findings, hemodynamics, and inflammatory variants. The results suggest that repeated LS application in advanced HF is advantageous when compared to single-dose LS administration.

\section{Acknowledgements}

The authors would like to thank Prof. Dr. Esmeray Acarturk for all her input and guidance on this manuscript.

2. Packer M, Carver JR, Rodeheffer RJ et al: Effect of oral milrinone on mortality in severe chronic heart failure. The PROMISE Study Research Group. N Engl J Med, 1991; 325: 1468-75 
3. O'Connor CM, Gattis WA, Uretsky BF et al: Continuous intravenous dobutamine is associated with an increased risk of death in patients with advanced heart failure: insights from the Şolan International Randomized Survival Trial (FIRST). Am Heart J, 1999; 138: 78-86

4. Metra M, Ponikowski P, Dickstein K et al: Advanced chronic heart failure: a position statement from the Study Group on Advanced Heart Failure of the Heart Failure Association of the European Society of Cardiology. Eur J Heart Fail, 2007; 9: 684-94

5. Kasikcioglu HA, Cam N: Intravenous positive inotropic therapy for acute decompensated heart failure. Arch Turk Soc Cardiol, 2006; 34: 316-22

6. Sorsa T, Heikkinen S, Abbott MB et al: Binding of levosimendan, a calcium sensitizer, to cardiac troponin C. J Biol Chem, 2001; 276: 9337-43

7. Nieminen MS, Akkila J, Hasenfuss G et al: Hemodynamic and neurohumoral effects of continuous infusion of levosimendan in patients with congestive heart failure. J Am Coll Cardiol, 2000; 36: 1903-12

8. Moiseyev VS, Põder P, Andrejevs $\mathrm{N}$ et al: Safety and efficacy of a novel calcium sensitizer, levosimendan, in patients with left ventricular failure due to an acute myocardial infarction. A randomized, placebo-controlled, double-blind study (RUSSLAN). Eur Heart J, 2002; 23: 1422-32

9. Follath F, Cleland JG, Just $\mathrm{H}$ et al: Efficacy and safety of intravenous levosimendan compared with dobutamine in severe low-output heart failure (the LIDO study): a randomised double-blind trial. Lancet, 2002; 360: 196-202

10. Francis G: Pathophysiology of chronic heat failure. Am J Med, 2001; 110: 37-46

11. Maeda K, Tsutamoto T, Wada A et al: High levels of plasma brain natriuretic peptide and interleukin- 6 after optimized treatment for heart failure are independent risk factors for morbidity and mortality in patients with congestive heart failure. J Am Coll Cardiol, 2000; 36: 1587-93

12. Parissis JT, Adamopoulos S, Farmakis D et al: Effects of serial levosimendan infusions on left ventricular performance and plasma biomarkers of myocardial injury and neurohormonal and immune activation in patients with advanced heart failure. Heart, 2006; 92: 1768-72

13. Shiller NB: Two-dimensional echocardiographic determination of left ventricular volume, sistolic function and mass. Circulation, 1991; 84: 1280-87

14. Mosterd A, Hoes AW, de Bruyne MC et al: Prevalence heart failure and left ventricular dysfunction in the general population. Eur Heart J, 1999; 20: 447-55

15. Reitsma JB, Mosterd A, de Craen AJ et al: Increase in hospitalization rates for heart failure in the Netherlands, 1980-1993. Heart, 1996; 76: 388-92

16. Shiba N, Nochioka K, Miura M et al: Trend of westernization of etiology and clinical characteristics of heart failure patients in Japan: First report from the CHART-2 study. Circ J, 2011; 75: 823-33

17. Greenberg B: Acute decompensated heart failure - treatments and challenges. Circ J, 2012; 76: 532-43

18. Mann DL: Mechanisms and models in heart failure: a combinatorial approach. Circulation, 1999; 100: 999-1008

19. Sasayama S, Matsumori A, Kihara Y: New insights into the pathophysiolog ical role for cytokines in heart failure. Cardiovasc Res, 1999; 42: 557-64

20. Seta Y, Shan K, Bozkurt B et al: Basic mechanisms in heart failure: the cytokine hypothesis. J Card Fail, 1996; 3: 243-49
21. Maeda K, Tsutamoto T, Wada A et al: High levels of plasma brain natriuretic peptide and interleukin- 6 after optimized treatment for heart failure are independent risk factors for morbidity and mortality in patients with congestive heart failure. J Am Coll Cardiol, 2000; 36: 1587-93

22. Cheng V, Kazanagra R, Garcia A et al: A rapid bedside test for B-type peptide predicts treatment outcomes in patients admitted for decompensated heart failure: a pilot study. J Am Coll Cardiol, 2001; 37: 386-91

23. Moertl D, Berger R, Huelsmann $M$ et al: Short-term effects of levosimendan and prostaglandin E1 on hemodynamic parameters and B-type natriuretic peptide levels in patients with decompensated chronic heart failure. Eur J Heart Fail, 2005; 7: 1156-63

24. Avgeropoulou C, Andreadou I, Markantonis-Kyroudis S et al: The $\mathrm{Ca}^{2+}$ sensitizer levosimendan improves oxidative damage, BNP and pro-inflammatory cytokine levels in patients with advanced decompensated heart failure in comparison to dobutamine. Eur J Heart Fail, 2005; 7: 882-87

25. Chatterjee K, Wolfe CL, De Marco T: Nonglycoside inotropes in congestive heart failure: are they beneficial or harmful? Cardiol Clin, 1994; 12: 63-72

26. Remondino A, Kwon SH, Communal C et al: Beta-adrenergic receptor-stimulated apoptosis in cardiac myocytes is mediated by reactive oxygen species/ c-Jun NH2- terminal kinase-dependent activation of the mitochondrial pathway. Circ Res, 2003; 92: 136-38

27. Hasenfuss G, Pieske B, Castell M et al: Influence of the novel inotropic agent levosimendan on isometric tension and calcium cycling in failing human myocardium. Circulation, 1998; 98: 2141-47

28. Papp Z, Csapó K, Pollesello P et al: Pharmacological mechanisms contributing to the clinical efficacy of levosimendan. Cardiovasc Drug Rev, 2005. 23: 71-98

29. Figgitt DP, Gillies PS, Goa KL: Levosimendan. Drugs, 2001; 61: 613-27

30. Michaels AD, McKeown B, Kostal M et al: Effects of intravenous levosimendan on human coronary vasomotor regulation, left ventricular wall stress, and myocardial oxygen uptake. Circulation, 2005; 111: 1504-9

31. Innes CA, Wagstaff AJ: Levosimendan: a review of its use in the management of acute decompensated heart failure. Drugs, 2003; 63: 2651-71

32. Earl GL, Fitzpatrick JT: Levosimendan: a novel inotropic agent for treatment of acute, decompensated heart failure. Ann Pharmacother, 2005; 39: 1888-96

33. Leier CV, Binkley PF: Parenteral inotropic support for advanced congestive heart failure. Prog Cardiovasc Dis, 1998; 41: 207-24

34. Kivikko M, Lehtonen L, Colucci WS: Sustained hemodynamic effects of intravenous levosimendan. Circulation, 2003; 107: 81-86

35. De Luca L, Colucci WS, Nieminen MS et al: Evidence-based use of levosimendan in different clinical settings. Eur Heart J, 2006; 27: 1908-20

36. Mavrogeni S, Giamouzis G, Papadopoulou E et al: A 6-month follow-up of repeated levosimendan administration effects on systolic function, specific activity questionnaire, and arrhythmia in advanced heart failure. J Card Fail, 2007; 13: 556-59

37. Dernellis J, Panaretou M: Effects of levosimendan on restrictive left ventricular filling in severe heart failure: a combined hemodynamic and Doppler echocardiographic study. Chest, 2005; 128: 2633-39

38. Kasikcioglu HA, Unal S, Tartan Z et al: Effects of levosimendan on left ventricular functional remodelling and exercise intolerance: a tissue Doppler study. J Int Med Res, 2005; 33: 397-405 